\title{
Medial and Lateral Discoid Menisci of Both Knees
}

\author{
Hiroyuki Kan, MD, Yuji Arai, MD, Shuji Nakagawa, MD, Hiroaki Inoue, MD, Ginjiro Minami, MD, \\ Kazuya Ikoma, MD, Hiroyoshi Fujiwara, MD, and Toshikazu Kubo, MD \\ Department of Orthopaedics, Graduate School of Medical Science, Kyoto Prefectural University of Medicine, Kyoto, Japan
}

\begin{abstract}
Discoid menisci on both the medial and lateral sides are rare, and there are very few reports on cases involving both sides. We report a case of a 52-year-old female with medial and lateral discoid menisci in both knees. Arthroscopy revealed the lateral menisci of both knees were complete discoid menisci, and partial meniscectomy was performed. The medial menisci were incomplete discoid menisci, but there were no findings of abnormal mobility or injury; therefore, the medial menisci were observed without treatment. At six months postoperatively, her pain and range of motion restrictions disappeared.
\end{abstract}

Keywords: Knee, Meniscus, Discoid, Medial, Lateral

The discoid meniscus is a malformation frequently observed in the lateral meniscus and thought to be more common in Asians than in other ethnicities ${ }^{1)}$. However, the medial discoid meniscus is rare, with a prevalence of $0.03 \%-0.3 \%$, and is even less common in both knees (range, $0.01 \%$ to $0.03 \%)^{2,3)}$. This report describes a patient with medial and lateral discoid menisci in both knees. The patient was informed that data from the case would be submitted for publication, and she gave her consent. Ethical approval for this study was obtained from the Ethical Review Board of our hospital.

\section{Case Report}

A 52-year-old female experienced right knee joint pain when going up and down stairs one month prior to her presentation to a clinic. Examination by a local doctor and magnetic resonance

Received November 24, 2015; Revised (1st) December 28, 2015;

(2nd) January 27, 2016; Accepted February 3, 2016

Correspondence to: Yuji Arai, MD

Department of Orthopaedics, Graduate School of Medical Science,

Kyoto Prefectural University of Medicine, 465 Kajiicho, Kawaramachi-

Hirokoji, Kamigyo-ku, Kyoto 602-8566, Japan

Tel: +81-75-251-5549, Fax: +81-75-251-5841

E-mail: yarai89046@nike.eonet.ne.jp

This is an Open Access article distributed under the terms of the Creative Commons Attribution Non-Commercial License (http://creativecommons.org/licenses/by-nc/4.0/) which permits unrestricted non-commercial use, distribution, and reproduction in any medium, provided the original work is properly cited. imaging (MRI) resulted in a diagnosis of lateral meniscus injury. She was treated conservatively for 2 months, but the pain was not alleviated, and her left knee joint also became painful so she was referred to our hospital immediately. Examination at our department showed a restriction in knee joint range of motion (ROM), with right knee extension $-15^{\circ}$ and flexion $125^{\circ}$ and left knee extension $-10^{\circ}$ and flexion $135^{\circ}$. Tenderness was present in the lateral femorotibial joint spaces of both knees, and clicking was palpated during flexion. The McMurray test also revealed lateral pain and clicking in both knees. There were no indirect

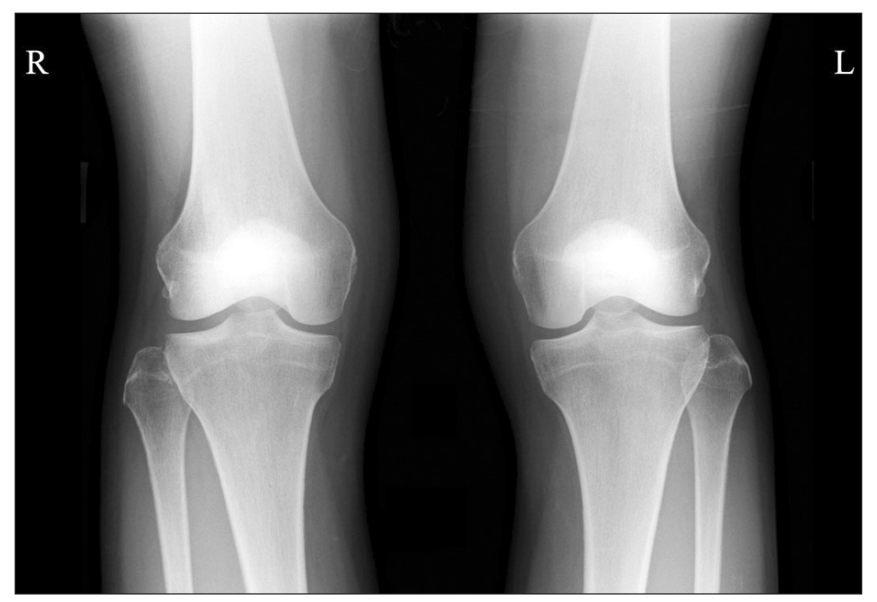

Fig. 1. Plain radiograph showing hypoplasia of the lateral femoral condyle and varus deformity of the articular surface of the proximal tibia. R: right, L: left. 


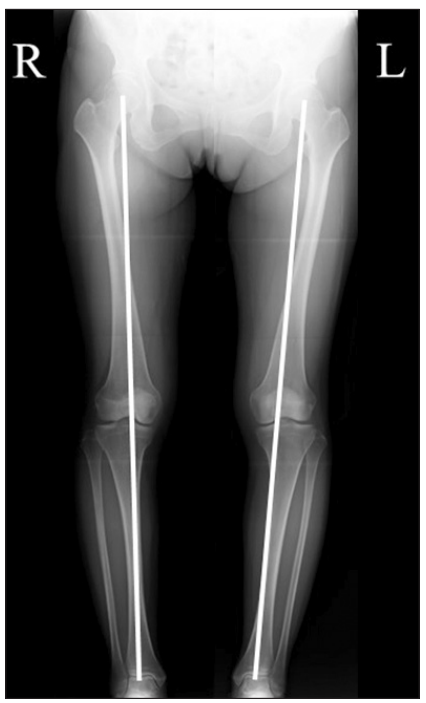

Fig. 2. Full-length frontal plain radiograph revealing hypoplasia of the lateral femoral condyle and varus deformity of the articular surface of the proximal tibia. Both Mikulicz lines were slightly displaced inwardly. R: right, L: left. signs of discoid lateral meniscus such as lateral joint space widening, squaring of the lateral femoral condyle, high fibular head, or hypoplasia of the lateral tibial plateau in plain radiographs (Fig. 1). Full-length frontal plain radiographs of the legs showed hypoplasia of the femoral lateral condyle and varus deformity of the articular surface of the proximal tibia. The femorotibial angle was $176^{\circ}$ on the right side and $177^{\circ}$ on the left side. The mechanical lateral distal femoral angle was $83^{\circ}$ on the right side and $80^{\circ}$ on the left side. The medial proximal tibial angle was $80^{\circ}$ on the right side and $77^{\circ}$ on the left side. Both Mikulicz lines were slightly displaced inwardly (Fig. 2) ${ }^{4}$. T2-weighted MRI showed that the medial menisci in both knees were incomplete discoid menisci, and there was high intensity in the posterior section. T2-weighted MRI of the lateral menisci in both knees showed complete discoid menisci and a horizontal high intensity area (Fig. 3). She underwent surgery immediately. Arthroscopy revealed that the lateral menisci of both knees were complete discoid menisci. Based on these findings, the patient underwent partial
$\mathrm{R}$

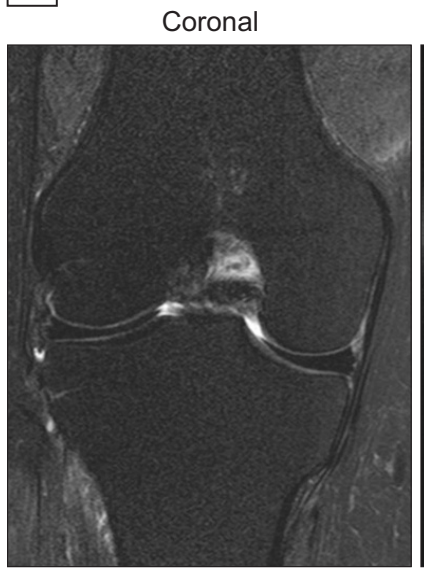

T2-star $\mathrm{L}$

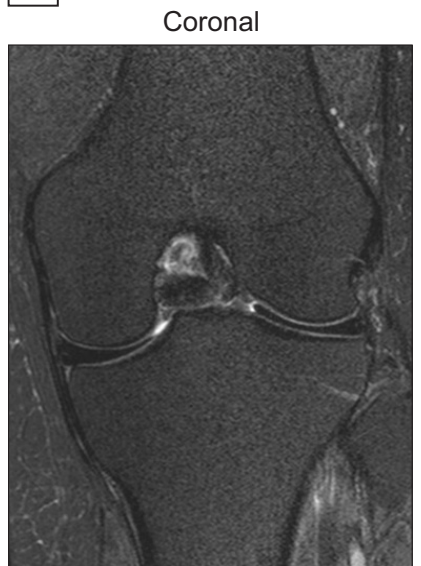

T2-star

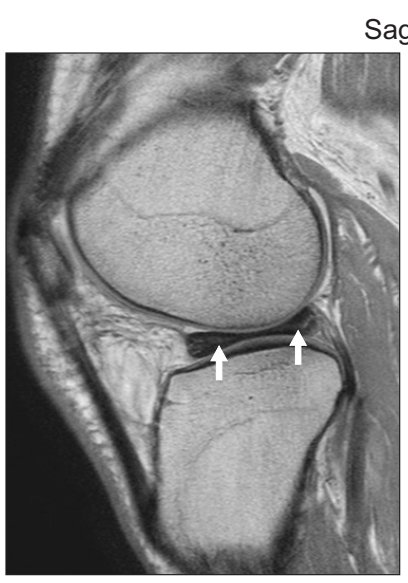

Lateral
Sagittal
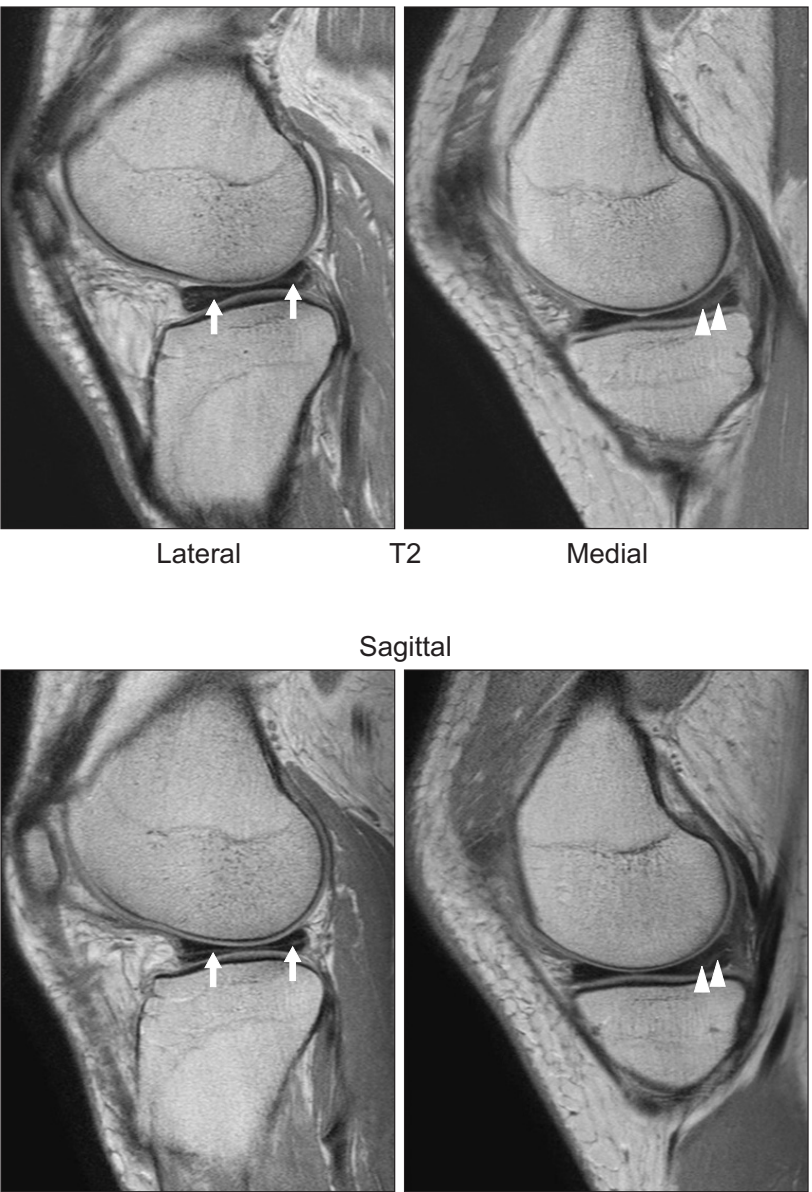

Lateral
Sagittal

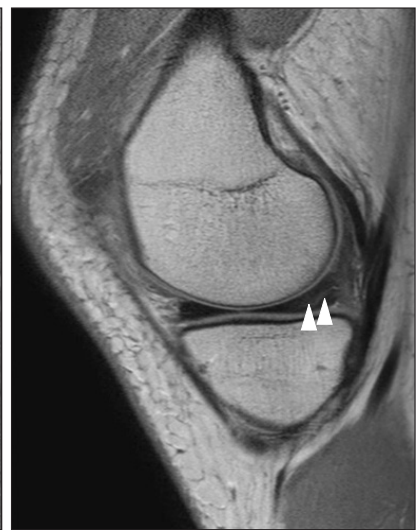

Medial
Fig. 3. T2-weighted magnetic resonance imaging revealed the medial menisci of both knees were incomplete discoid menisci with a high intensity area in the posterior section (arrowheads). The lateral menisci were complete discoid menisci with a horizontal high intensity area (arrows). R: right, L: left. 


\section{Kan et al. Medial and Lateral Discoid Menisci of Both Knees}

$\mathrm{R}$

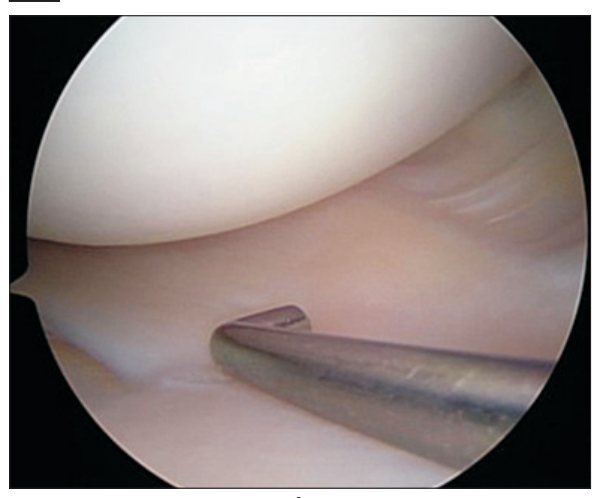

A
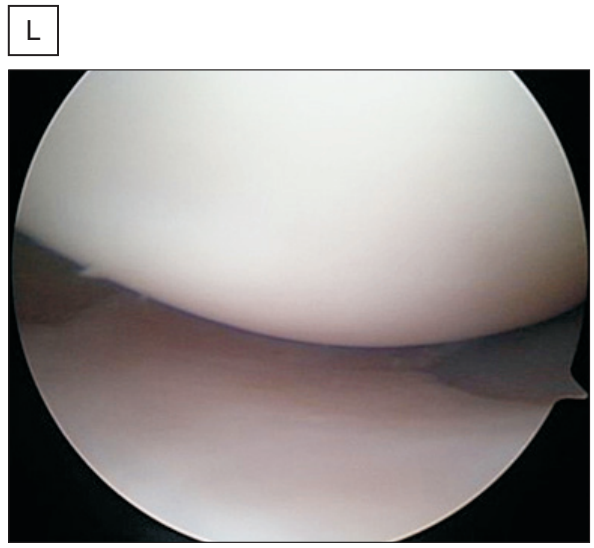

A

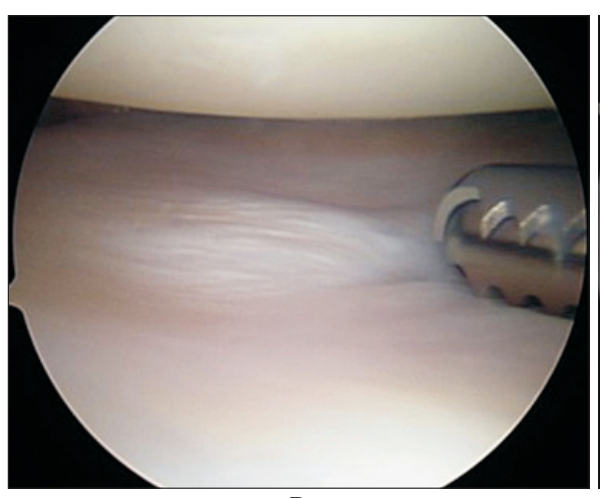

B

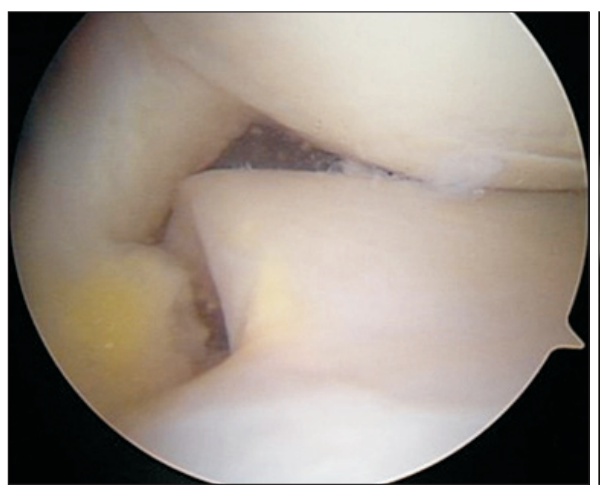

B

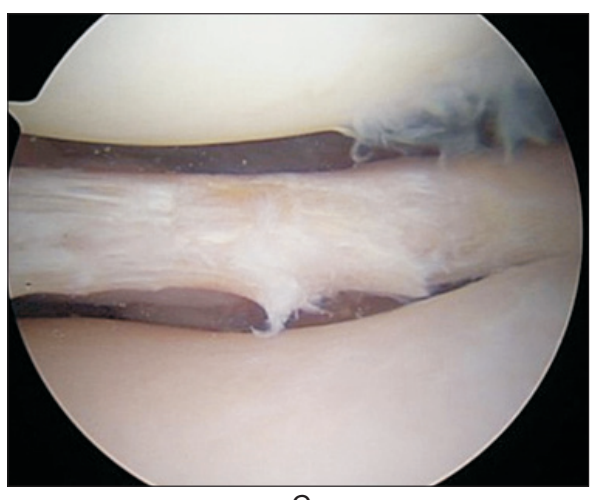

C

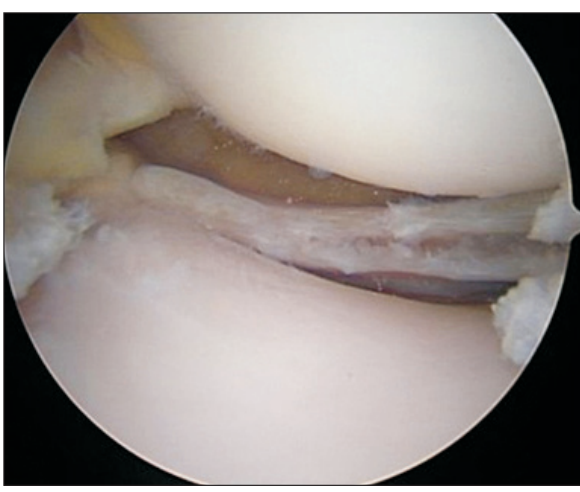

C

Fig. 4. Arthroscopic findings of both knees. The medial menisci were incomplete discoid menisci, but there were no findings of abnormal mobility or injury (A). The lateral menisci were complete discoid menisci (B) and partial meniscectomy was performed, during which a horizontal tear (C) was observed from the anterior to the posterior section. R: right, L: left.

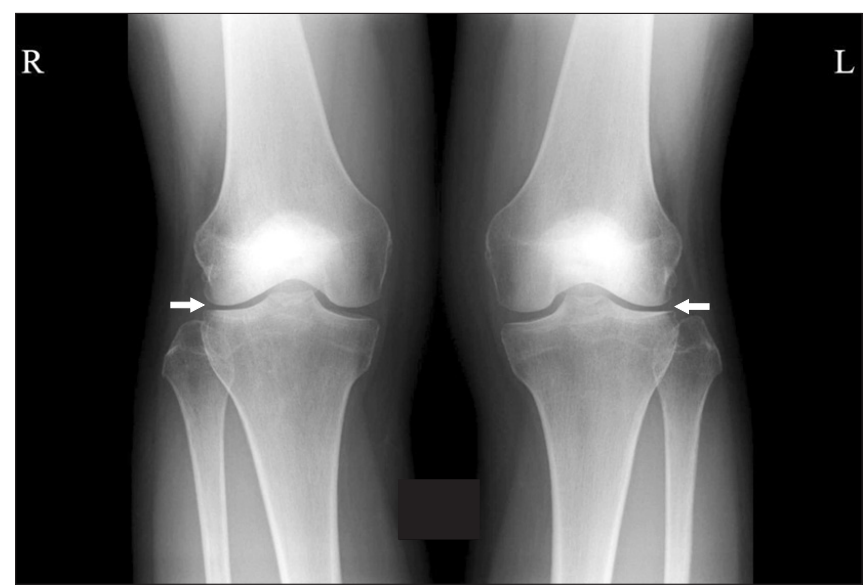

Fig. 5. Plain radiograph showing lateral femorotibial joint space narrowing (arrows) at 1 year and 7 months after surgery. R: right, L: left.

meniscectomy, leaving margins of about $6 \mathrm{~mm}$. There was a horizontal tear from the anterior to the posterior section. The medial menisci were incomplete discoid menisci, but there were no find- ings of abnormal mobility or injury; therefore, and the medial menisci were observed without treatment (Fig. 4). At six months postoperatively, her pain and ROM restrictions disappeared. MRI showed no obvious changes in the medial menisci. One year and seven months postoperatively, she experienced no recurrence of pain or hydrarthrosis, and plain X-rays showed lateral femorotibial joint space narrowing (Fig. 5).

\section{Discussion}

The discoid meniscus is an anatomical malformation of the meniscus thought to be more common in Asians. The reported prevalence of lateral discoid meniscus is $3.0 \%-5.0 \%$, with $81.5 \%$ $97.0 \%$ of these patients having bilateral lateral discoid menisci ${ }^{5)}$. In contrast, medial discoid menisci, first reported in $1941^{6}$, is rare, with a prevalence of $0.03 \%-0.3 \%$, and is even less common in both knees (range, $0.01 \%$ to $0.03 \%)^{2,3}$. Tissue strength has been reported to differ in the lateral and medial menisci ${ }^{7)}$, mak- 
ing the medial meniscus more susceptible to cracks in the center during the embryonic stage and discoid meniscus less likely to occur.

Although clinical symptoms of clicking, locking, and swelling are thought to be common, none of these is characteristic, and MRI is essential for an accurate diagnosis. Similar to the lateral discoid meniscus, the medial discoid meniscus is suspected when three or more 5-mm slices of sagittal images show continuity of the anterior to the posterior sections of the medial menisci, or when coronal images show a transverse diameter of $\geq 15 \mathrm{~mm}^{8}$. In the present patient, sagittal images showed continuity in the medial menisci on seven 2.2-mm slices and in the lateral menisci on ten slices. In addition, coronal images showed a transverse diameter of $\geq 15 \mathrm{~mm}$ in the medial menisci and $\geq 22 \mathrm{~mm}$ in the lateral menisci of both knees.

Compared with normal menisci, discoid menisci are thought to be more easily injured. This patient showed abnormal intensities in a substantial portion of both the medial and lateral menisci on MRI, but they were more severe on the lateral side. Mechanical stress was more likely to be placed on the lateral side, since it was a complete discoid meniscus, whereas the medial side was incomplete, resulting in injury only to the lateral side.

The surgical indications for discoid meniscus on the medial side are unclear because of the small number of patients hitherto described. On the lateral side, it has been recommended that patients be observed if there is no injury. Arthroscopic meniscectomy and repair are the surgical options for symptomatic lateral discoid menisci, and good results have been reported ${ }^{9)}$. Repair outcomes have been reported similarly for both lateral and medial menisci ${ }^{10)}$, and it has been recommended that the discoid meniscus be preserved if severe symptoms are not present. The patient described here played sports recreationally, but experienced only lateral pain and clicking in both knees. MRI showed a horizontal high intensity area in the complete discoid lateral menisci, whereas a high intensity area was seen in the posterior section of the medial menisci. In addition, arthroscopic findings of injury were seen on the lateral side, whereas there were no findings of abnormal mobility or injury on the medial side. Therefore, in this patient, only the lateral menisci were resected, while the medial menisci were left intact. This surgery resulted in the disappearance of pain and ROM restrictions. This patient has experienced a good clinical course to date, although the duration of follow-up has been short. A longer follow-up period is required since the discoid meniscus has been reported to be a risk factor for articular cartilage lesions.
This report describes a very rare case of medial and lateral discoid menisci in both knees. Partial meniscectomy was performed on the lateral menisci only based on the clinical and imaging findings of meniscal injury. The patient demonstrated a good postoperative course but requires a long-term follow-up.

\section{Conflict of Interest}

No potential conflict of interest relevant to this article was reported.

\section{References}

1. Ahn JH, Lee YS, Ha HC, Shim JS, Lim KS. A novel magnetic resonance imaging classification of discoid lateral meniscus based on peripheral attachment. Am J Sports Med. 2009;37:1564-9.

2. Marchetti ME, Jones DC, Fischer DA, Boyd JL, Fritts HM. Bilateral discoid medial menisci of the knee. Am J Orthop (Belle Mead NJ). 2007;36:317-21.

3. Kim SJ, Lubis AM. Medial and lateral discoid menisci: a case report. Sports Med Arthrosc Rehabil Ther Technol. 2010;2:21.

4. Paley D. Normal lower limb alignment and joint orientation. In: Paley D, ed. Principles of deformity correction. Berlin: Springer; 2002. p1-18.

5. Ahn JH, Lee SH, Yoo JC, Lee HJ, Lee JS. Bilateral discoid lateral meniscus in knees: evaluation of the contralateral knee in patients with symptomatic discoid lateral meniscus. Arthroscopy. 2010;26:1348-56.

6. Cave EF, Staples OS. Congenital discoid meniscus: a cause of internal derangement of the knee. Am J Surg. 1941;54:371-6.

7. Fukazawa I, Hatta T, Uchio Y, Otani H. Development of the meniscus of the knee joint in human fetuses. Congenit Anom (Kyoto). 2009;49:27-32.

8. Scott WN. Surgery of the Knee. 4th ed. Philadelphia, PA: Churchill Livingstone/Elsevier; 2006. p1223-33.

9. Ahn JY, Kim TH, Jung BS, Ha SH, Lee BS, Chung JW, Kim JM, Bin SI. Clinical results and prognostic factors of arthroscopic surgeries for discoid lateral menisci tear: analysis of 179 cases with minimum 2 years follow-up. Knee Surg Relat Res. 2012;24:108-12.

10. Kraus T, Heidari N, Svehlik M, Schneider F, Sperl M, Linhart W. Outcome of repaired unstable meniscal tears in children and adolescents. Acta Orthop. 2012;83:261-6. 